\title{
Higher neutrophil-to-lymphocyte ratio after the first cycle of the first-line chemotherapy is associated with poor cancer specific survival of upper urinary tract carcinoma patients
}

\author{
Mamoru Hashimoto^, Kazutoshi Fujita, Takahito Nakayama, Saizo Fujimoto, Mamoru Hamaguchi, \\ Mitsuhisa Nishimoto, Takashi Kikuchi, Shogo Adomi, Eri Banno, Marco A. De Velasco, Yoshitaka Saito, \\ Nobutaka Shimizu, Yasunori Mori, Takafumi Minami, Masahiro Nozawa, Kazuhiro Nose, \\ Kazuhiro Yoshimura, Hirotsugu Uemura
}

Department of Urology, Kindai University Faculty of Medicine, Osaka, Japan

Contributions: (I) Conception and design: K Fujita, M Hashimoto; (II) Administrative support: T Kikuchi, E Banno, T Minami, M Nozawa; (III) Provision of study materials or patients: K Yoshimura, H Uemura; (IV) Collection and assembly of data: M Nishimoto, K Nose, S Adomi, T Nakayama, S Fujimoto, M Hamaguchi, Y Saito; (V) Data analysis and interpretation: N Shimizu, Y Mori, MA De Velasco; (VI) Manuscript writing: All authors; (VII) Final approval of manuscript: All authors.

Correspondence to: Kazutoshi Fujita. Department of Urology, Kindai University Faculty of Medicine, 377-2, Ohno-Higashi, Osaka-Sayama, Osaka 589-8511, Japan. Email: kfujita@med.kindai.ac.jp. Background: Inflammatory cytokines and immature myeloid derived suppressor cells (MDSCs), which
increase during cancer progression, could lead to a neutrophil increase and lymphocyte reduction. Thus, the
neutrophil-lymphocyte ratio (NLR) was used to predict survival of patients suffering from urological cancers
including upper urinary tract carcinoma. We further determined whether the NLR during the first cycle of
first-line chemotherapy could predict cancer specific survival.
Methods: We recruited patients with locally advanced or metastatic upper urinary tract urothelial
carcinoma (UTUC) who received chemotherapy between January 2014 and July 2019 . We investigated the
impact of various clinical variables, including age, sex, performance status, and estimated creatinine clearance
(CCr), and NLR before and after the first cycle of the first-line chemotherapy on prognosis.
Results: A total of 41 patients were included in our study. Cancer specific survival of the patients with
lower NLR was significantly better than that of the patients with higher NLR measured after the first cycle
of the first-line chemotherapy (log-rank test P=0.005, median 29.2 vs. 11.9 months, respectively). Cox
proportional regression analysis showed that higher NLR after the first cycle of the first-line chemotherapy
was a significant predictor of cancer specific survival.
Conclusions: The NLR after the first cycle of the first-line chemotherapy could be an indication for
patients with locally advanced or metastatic UTUC to maintain their first-line chemotherapy treatment.

Keywords: Neutrophil; lymphocyte; neutrophil-lymphocyte ratio (NLR); upper urinary tract urothelial carcinoma (UTUC); chemotherapy

Submitted Mar 08, 2021. Accepted for publication May 19, 2021.

doi: $10.21037 /$ tau-21-185

View this article at: http://dx.doi.org/10.21037/tau-21-185

^ ORCID: 0000-0002-4137-942X. 


\section{Introduction}

Upper urinary tract urothelial carcinoma (UTUC) accounts for $5-10 \%$ of all urothelial carcinomas. This rare disease is often advanced at diagnosis and is associated with a poor prognosis (1-3). Hence, having an efficient biomarker which could predict survival in UTUC on treatment is important $(4,5)$. The neutrophil-lymphocyte ratio (NLR) can be easily calculated with a blood test, and this inflammatory marker is reported as prognosticator in urothelial carcinoma $(6,7)$. Locally advanced or metastatic bladder cancer patients with persistently high NLR, even after the first cycle of first-line chemotherapy experienced short term survival rates (7). UTUC is the same urothelial carcinoma as bladder cancer, but UTUC differs from bladder cancer in its responsiveness to chemotherapy (8). A higher NLR prior to chemotherapy predicted poor prognosis in patients with metastatic UTUC (6). However, studies that examine the association between persistently high NLR after the first cycle of first-line chemotherapy and prognosis in UTUC do not exist. In this study, we elucidated whether NLR during the first cycle of first-line chemotherapy could predict prognosis in locally advanced or metastatic (advanced) UTUC.

We present the following article in accordance with the REMARK reporting checklist (available at http://dx.doi. org/10.21037/tau-21-185).

\section{Methods}

The study was conducted in accordance with the Declaration of Helsinki (as revised in 2013). The study was approved by the institutional ethical review board (No. R02-247) and individual consent for this retrospective analysis was waived. UTUC patients who started their treatment between January 2014 and July 2019 were entered into this retrospective study. Inclusion criteria included patients with advanced UTUC, and they received at least one cycle of chemotherapy. Patients who received adjuvant chemotherapy after surgery, and had no recurrence thereafter were excluded. Patients with inflammatory disease including any infections were also excluded from the analysis. Blood test results were derived from the hematological analysis from two-time points, namely, before the first and second cycle of the first-line chemotherapy. We set a cutoff value for the NLR at three according to previous reports $(6,7)$. We divided the patients according to NLR. Patients with an NLR $<3$ and an NLR $\geq 3$ before the first cycle of the first-line chemotherapy were classified as "lower NLR (pre-chemo)" and "higher NLR (pre-chemo)" groups, respectively. Patients with an NLR $<3$ and an NLR $\geq 3$ after the first cycle of the first-line chemotherapy were classified as "lower NLR (post-chemo)" and "higher NLR (post-chemo)" groups, respectively. We performed cox proportional regression analysis to evaluate the impact of variables, including age, sex, performance status, and creatinine clearance (CCr), and NLR on cancer specific survival. The log-rank test was also performed to compare survival distributions between the two groups. CCr was estimated by the Cockcroft-Gault equation (9).

\section{Statistical analysis}

Statistical analyses were performed using EZR (Saitama Medical Center, Jichi Medical University, Saitama, Japan), which is a graphical user interface for $\mathrm{R}$ (The R Foundation for Statistical Computing, Vienna, Austria) (10). The association of variables between two groups was analyzed by the chi-square test, Fisher's exact test or Mann-Whitney U test. The paired $t$-test was used for the evaluation of NLR change. We performed log rank test and cox proportional regression analysis to evaluate variables which could affect survival rate. A P value $<0.05$ was considered as significant. Dot plots showing NLR distributions were created in GraphPad Prism version 8.00 for Windows, GraphPad Software, La Jolla, CA, USA, www.graphpad.com.

\section{Results}

A total of 41 patients were included in our study. Patient demographics are summarized in Table 1. No patients received neo-adjuvant chemotherapy before surgery. Nineteen patients received $1-3$ cycles of adjuvant chemotherapy. In this cohort, $21,17,4$, and 1 patient received Gemcitabine + Cisplatin, Gemcitabine + Carboplatin, Methotrexate + Vinblastine + Adriamycin + Cisplatin, and Carboplatin monotherapy as first-line chemotherapy, respectively. The median number of first-line chemotherapy cycles is 3 (range, 1-14). The combination of Gemcitabine and Cisplatin was effective for quite a long time in two patients. They received it intermittently for a total of 12 and 14 cycles, respectively. Six patients received pembrolizumab as second-line therapy. Median cancer specific survival was 17.2 months (95\% CI: 8.6-29.2). Twenty-six (63\%) patients died of tumor progression, $8(20 \%)$ patients were transferred to 
Table 1 The characteristics of all patients $(n=41)$, and associations of patient's characteristics between lower $(n=15)$ and higher $(n=26)$ pre-chemo NLR group ${ }^{\dagger}$

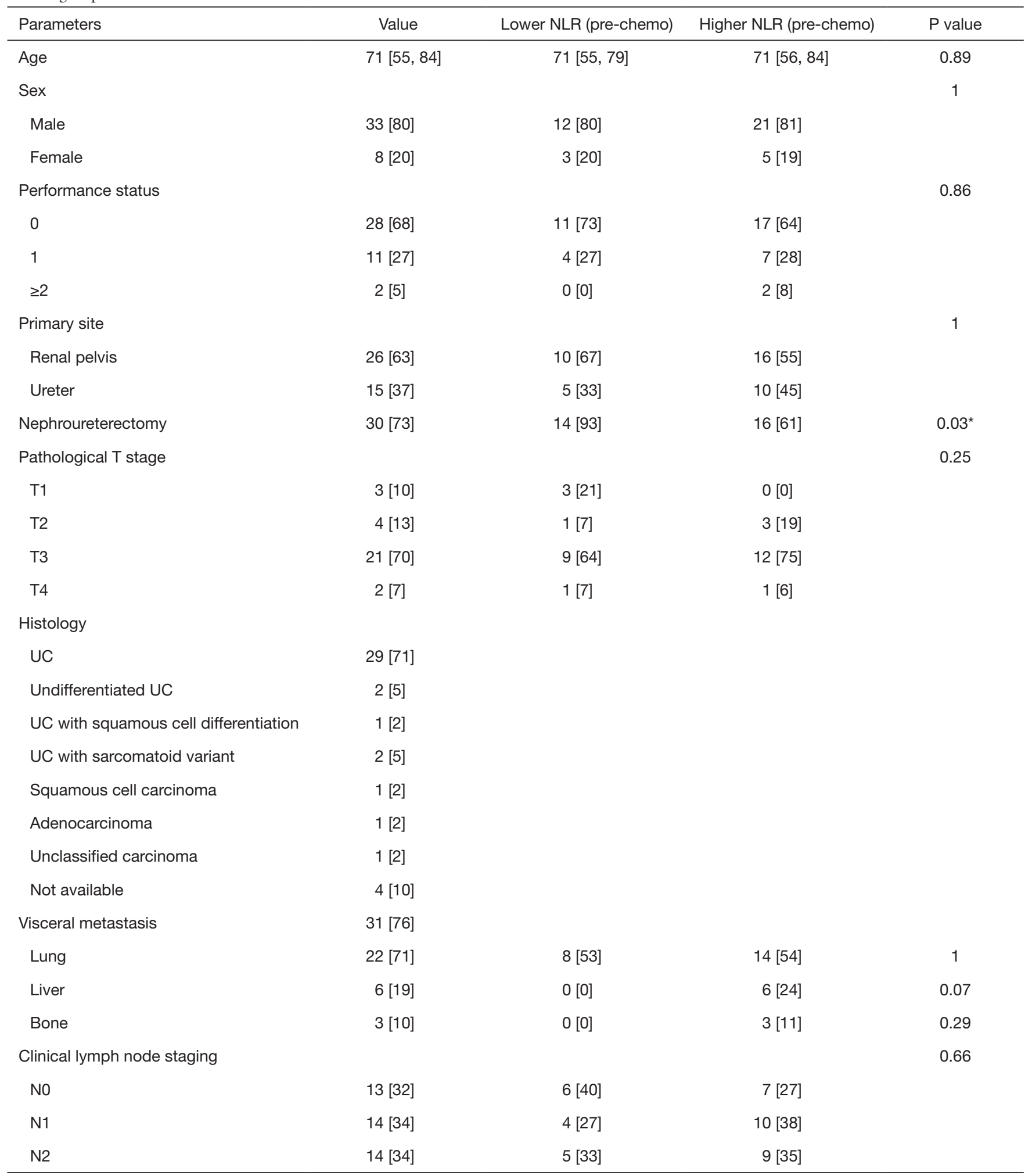

Table 1 (continued) 
Table 1 (continued)

\begin{tabular}{|c|c|c|c|c|}
\hline Parameters & Value & Lower NLR (pre-chemo) & Higher NLR (pre-chemo) & $P$ value \\
\hline $\mathrm{CCr}$ & $45.6[22.4,88.4]$ & $48.6[27.7,68.4]$ & $43.5[22.4,88.4]$ & 0.23 \\
\hline NLR & $3.61[1.96,7.50]$ & $2.29[1.96,2.94]$ & $4.31[3.07,7.50]$ & $<0.001^{*}$ \\
\hline \multicolumn{5}{|c|}{ NLR and CCr after the first cycle of the first-line chemotherapy } \\
\hline NLR & $3.16[0.67,29.5]$ & $2.32[0.66,5.60]$ & $3.47[0.98,29.5]$ & $0.006^{*}$ \\
\hline
\end{tabular}

Values represent number [\%], or median [range]. ${ }^{\dagger}$, patients with NLR $<3$ and an NLR $\geq 3$ before the first cycle of first-line chemotherapy were divided into lower NLR (pre-chemo) and higher NLR (pre-chemo) groups, respectively; *, a P value $<0.05$ was considered significant. NLR, neutrophil-lymphocyte ratio; UC, urothelial carcinoma; $\mathrm{CCr}$, creatinine clearance.

another hospital for hospice care because of severe disease progression, and 1 patient died from an unknown cause. We divided the patients according to the NLR, patients with an NLR $<3$ or an NLR $\geq 3$ before the first cycle of first-line chemotherapy were assigned to "lower NLR (pre-chemo)" and "higher NLR (pre-chemo)" groups, respectively. Patients with an NLR $<3$ or an NLR $\geq 3$ after the first cycle of first-line chemotherapy were assigned to "lower NLR (post-chemo)" and "higher NLR (postchemo)" groups, respectively. Statistically significant differences between lower NLR (pre-chemo) and higher NLR (pre-chemo) were observed in surgery and NLR (Table 1). There were no statistical differences in patient characteristics between lower NLR (post-chemo) and higher NLR (post-chemo) other than the NLR (Table 2). Overall, the NLR did not differ in patients during the first cycle of first-line chemotherapy (paired $t$-test, $\mathrm{P}=0.47$, Figure 1). Notably, the NLR decreased significantly during the first cycle of first-line chemotherapy in the lower NLR (post-chemo) cohort (paired $t$-test, $\mathrm{P}=0.0001$ ), whereas, the NLR did not change in the lower NLR (pre-chemo), higher NLR (pre-chemo) and higher NLR (post-chemo) groups. Cancer specific survival between lower NLR (prechemo) (median, 26.0 months) and higher NLR (prechemo) (median, 11.9 months) did not differ statistically (log-rank test, $\mathrm{P}=0.20$; Figure 2). However, cancer specific survival of the lower NLR (post-chemo) group (median, 29.2 months) was better than that of higher NLR (postchemo) (median, 11.9 months) (log-rank test, $\mathrm{P}=0.005$, Figure 3). Univariate and multivariate logistic regression analysis predicted which variables affected cancer specific survival. In blood tests performed before the first cycle of the first-line chemotherapy, univariate and multivariate analyses did not identify any variables as predictors of cancer specific survival (Table 3). On the other hand, in the blood test performed after the first cycle of the first-line chemotherapy revealed that on univariate analysis, NLR $(\mathrm{P}=0.007)$ was significantly associated with cancer specific survival and was still a significant predictor of cancer specific survival on multivariate analysis (model 1 : $\mathrm{P}=0.01$, model 2: $\mathrm{P}=0.03$, Table 4). Performance status, Ccr, pathological $\mathrm{T}$ stage, and surgery were not associated with CSS in this study, although there was a trend in histology (Tables 3,4).

\section{Supplementary result}

The patients were divided into four groups according to NLR change before and after the first cycle of first-line chemotherapy. The groups were named as high-low, highhigh, low-low, and low-high. The median CSS (month) of the patients in high-low, high-high, low-low, and lowhigh were NA [ $\mathrm{n}=8,95 \%$ CI: 6.57-not available (NA)], 8.57 ( $\mathrm{n}=18,95 \%$ CI: 4.57-16.7), 29.2 ( $\mathrm{n}=11,95 \%$ CI: 17.2-NA) and 18.7 ( $n=4,95 \%$ CI: 3.28-NA). Although the number of patients in each group was limited, the patients with lowlow and high-high seemed to show better and worse CSS, respectively.

\section{Discussion}

We demonstrated that NLR $\geq 3$ after the first cycle of firstline chemotherapy was a significant predictor of worse cancer specific survival. A previous article showed that locally advanced or metastatic bladder cancer patients with higher NLR after the first cycle of the first-line 
Table 2 Associations of patient's characteristics between lower $(\mathrm{n}=19)$ and higher $(\mathrm{n}=22)$ post-chemo NLR group ${ }^{\dagger}$

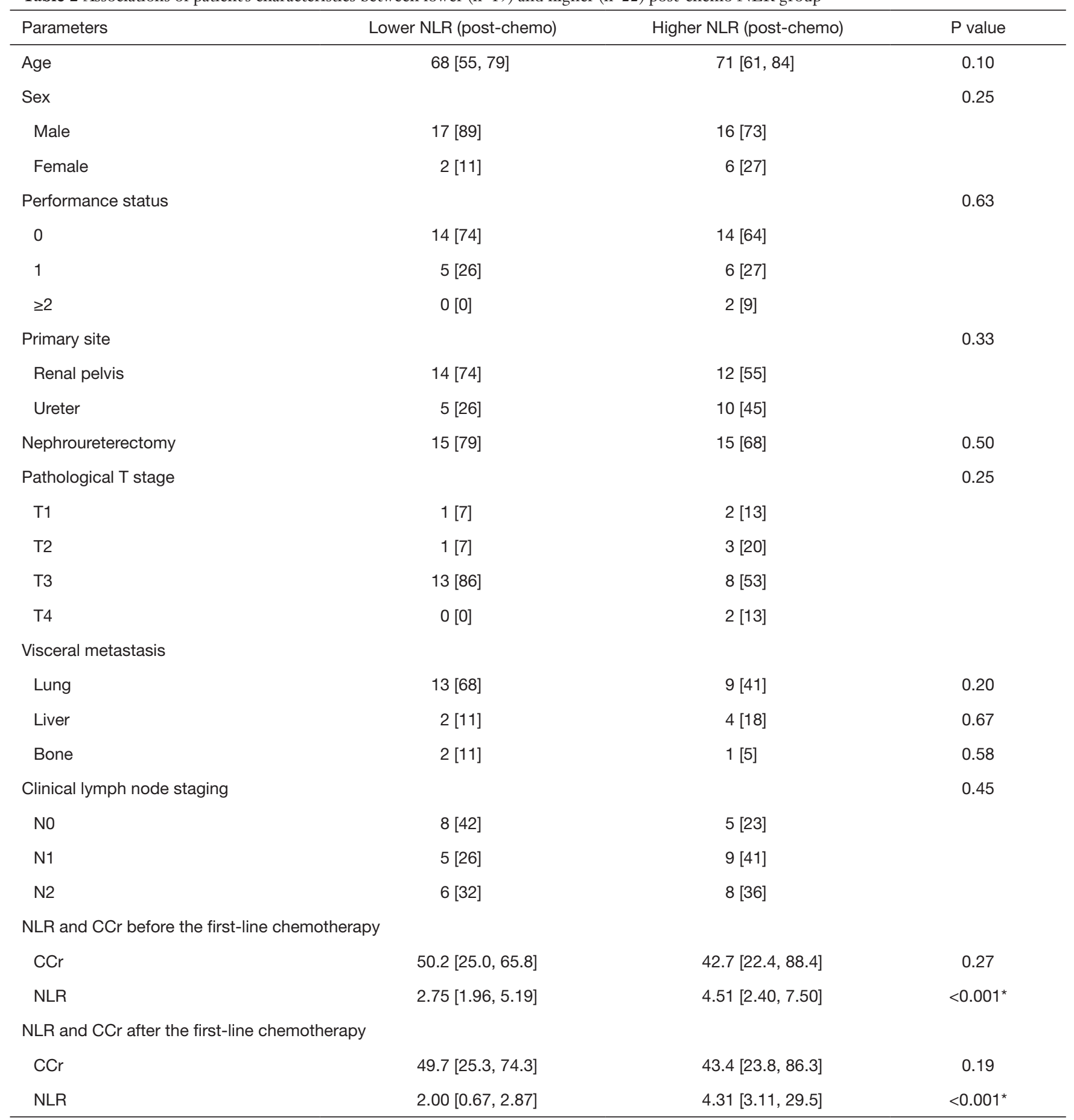

Values represent number [\%], or median [range]. ${ }^{\dagger}$, patients with an NLR $<3$ and an NLR $\geq 3$ after the first cycle of first-line chemotherapy were divided into lower NLR (post-chemo) and higher NLR (post-chemo) groups, respectively; *, a P value $<0.05$ was considered significant. NLR, neutrophil-lymphocyte ratio; CCr, creatinine clearance. 
chemotherapy had worse overall survival (7). Another study revealed that baseline NLR before the first-line chemotherapy could be a prognostic factor for the urothelial carcinoma including UTUC (6). However, it had not yet been elucidated whether a higher NLR after the first cycle of the first-line chemotherapy could be associated with prognosis in UTUC patients. Patients with UTUC often present with advanced stages of the disease at diagnosis (1). Furthermore, we sometimes encounter UTUC patients

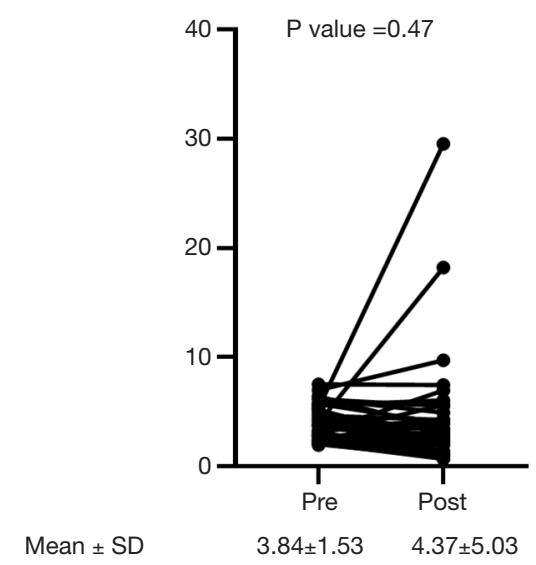

Figure 1 NLR change during the first cycle of first-line chemotherapy in all patients. The paired $t$-test was performed to compare NLR between pre- and post-first cycle of first-line chemotherapy. A P value $<0.05$ was considered significant. NLR, neutrophil-lymphocyte ratio. with renal impairment after nephroureterectomy, and a dose reduction in first-line platinum-based chemotherapy may affect outcomes (11). Thus, strong indicators for that allow for the identification of patients that should continue firstline chemotherapy are needed. A previous study indicated that the number of first-line chemotherapy cycles did not contribute to survival benefit in urothelial carcinoma patients (12). However, our study suggests that an NLR $\geq 3$ after the first cycle of the first-line chemotherapy could be indicative of patients that could continue first-line chemotherapy.

Neutrophils in cancers are referred to as tumorassociated neutrophils (TANs) and depending on the stage of tumor progression, they play dual roles as both tumor suppressors and promoters. During the early stages of progression TANs play key roles in antitumor immunity producing proinflammatory cytokines and infiltrating tumors. Moreover, TANs promote T-cell proliferation and interferon- $\gamma$ release. However, as cancers progress, TANs lose their tumoricidal capability and instead become tumorigenic. Cancer cells can also induce TANs to release inflammatory cytokines to acquire metastatic and invasive capabilities. Even worse, it is during these stages that immature myeloid-derived suppressor cells (MDSCs) also emerge. MDSCs are involved with immunosuppression, which disrupt T-cell proliferation, resulting in decreased antitumor immunity (13). Higher levels of MDSCs are associated with worse prognosis in metastatic urothelial carcinoma patients (14). Tumor neutrophilia is prompted

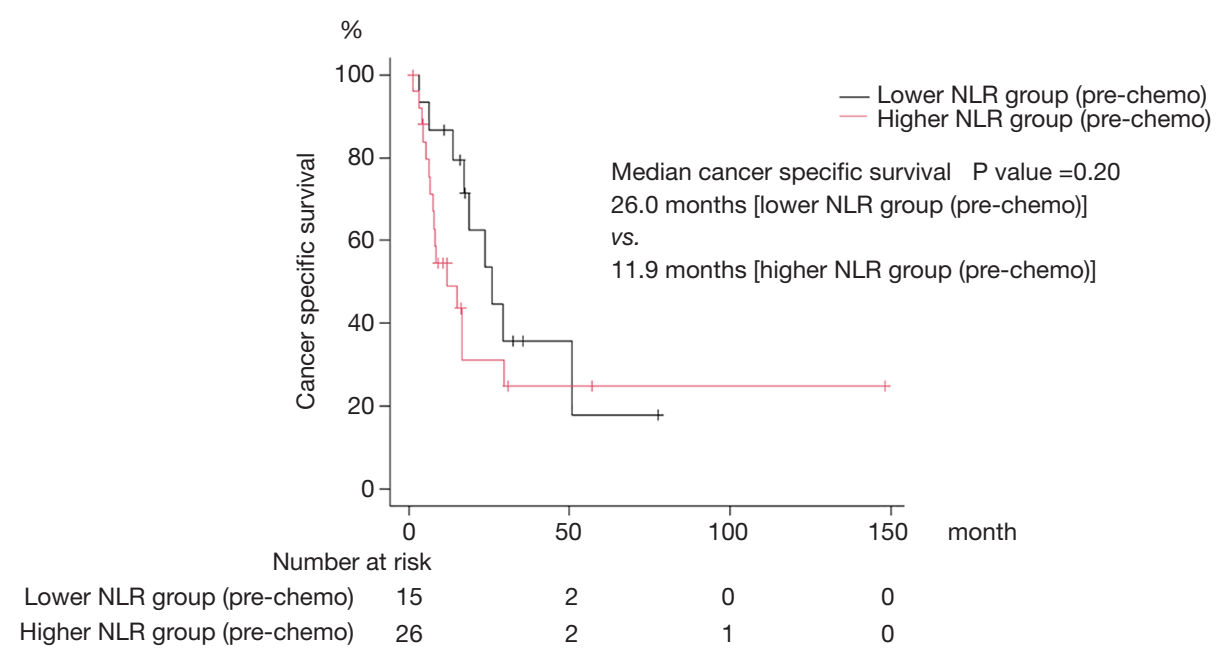

Figure 2 The log rank test was performed to compare survival distributions between lower NLR (pre-chemo) and higher NLR (pre-chemo). A P value $<0.05$ was considered significant. NLR, neutrophil-lymphocyte ratio. 


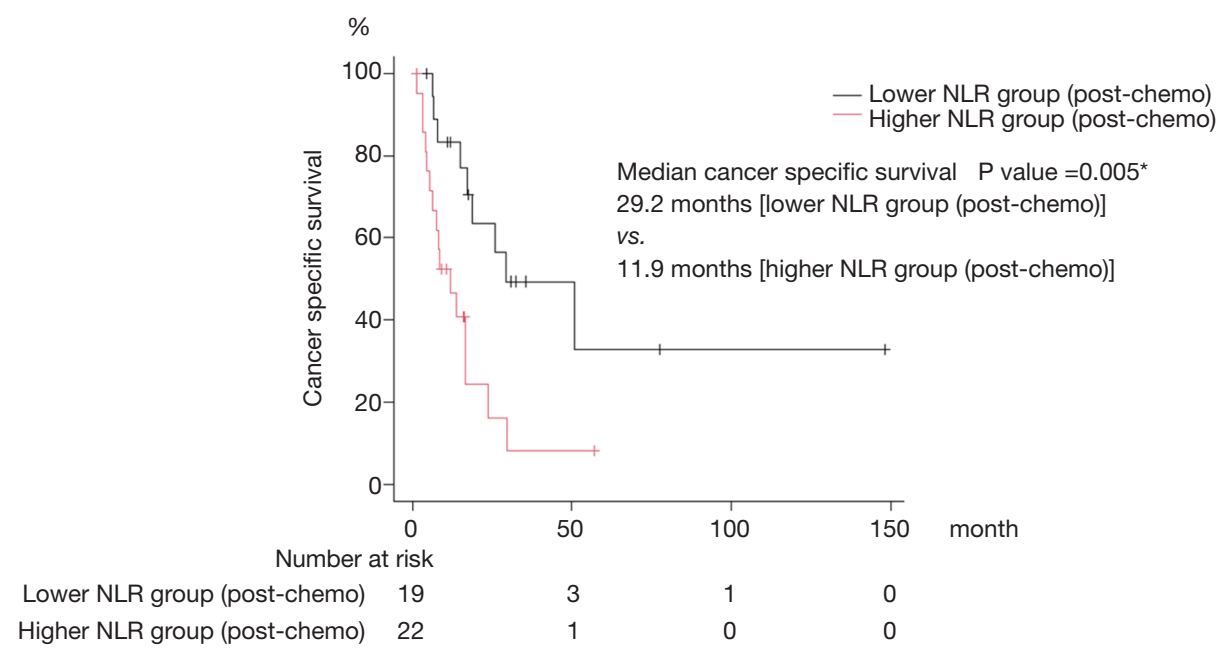

Figure 3 The log rank test was performed to compare survival distributions between lower NLR (post-chemo) and higher NLR (post-chemo). A P value $<0.05$ was considered significant. NLR, neutrophil-lymphocyte ratio.

Table 3 Cox proportional hazards regression analysis for cancer specific survival

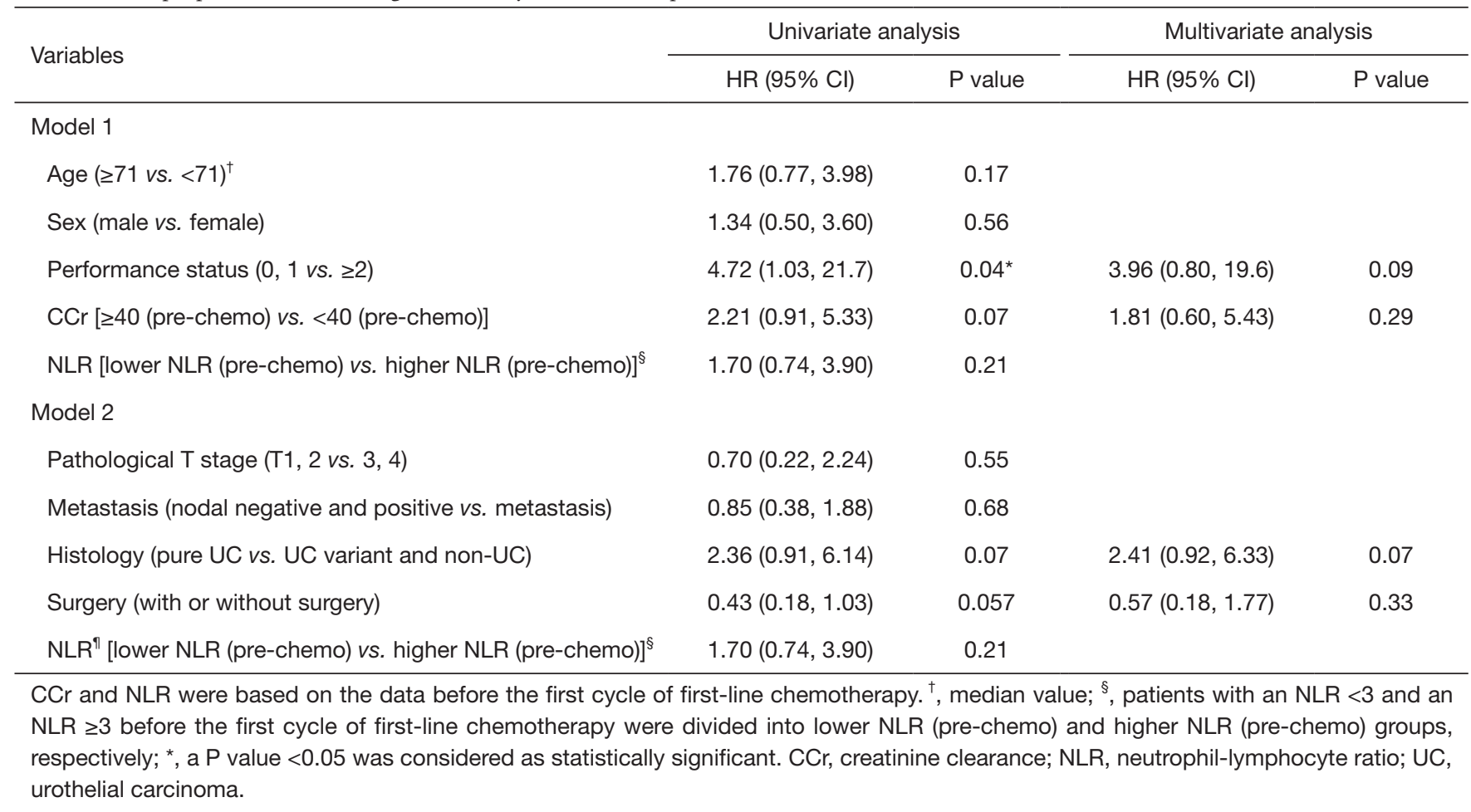

by tumor-generated cytokines, such as IL-6, granulocytecolony stimulating factor, and granulocyte macrophage colony-stimulating factor (13). Thus, we can presume that a higher NLR could be associated with worse prognosis, particularly in patients with advanced disease. However, evidence of the persistent increase of NLR even after chemotherapy being related with prognosis in UTUC is limited. Nevertheless, a higher NLR after the first cycle of the first-line chemotherapy could be associated with prognosis in bladder cancer patients (7). Another report 
Table 4 Cox proportional hazards regression analysis for cancer specific survival

\begin{tabular}{|c|c|c|c|c|}
\hline Variables & \multicolumn{2}{|c|}{ Univariate analysis } & \multicolumn{2}{|c|}{ Multivariate analysis } \\
\hline \multicolumn{5}{|l|}{ Model 1} \\
\hline Age $(\geq 71 \text { vs. }<71)^{\dagger}$ & $1.76(0.77,3.98)$ & 0.17 & & \\
\hline Sex (male vs. female) & $1.34(0.50,3.60)$ & 0.56 & & \\
\hline $\operatorname{CCr}[\geq 40$ (post-chemo) vs. $<40$ (post-chemo)] & $1.69(0.60,4.75)$ & 0.32 & & \\
\hline NLR [lower NLR (post-chemo) vs. higher NLR (post-chemo)] & $3.18(1.37,7.39)$ & $0.007^{*}$ & $2.98(1.25,7.05)$ & $0.01^{*}$ \\
\hline \multicolumn{5}{|l|}{ Model 2} \\
\hline Pathological T stage (T1, 2 vs. 3, 4) & $0.70(0.22,2.24)$ & 0.55 & & \\
\hline Surgery (with or without surgery) & $0.43(0.18,1.03)$ & 0.057 & $0.56(0.18,1.74)$ & 0.31 \\
\hline NLR" [lower NLR (post-chemo) vs. higher NLR (post-chemo)] & $3.18(1.37,7.39)$ & $0.007^{*}$ & $2.71(1.09,6.70)$ & $0.03^{*}$ \\
\hline
\end{tabular}

CCr and NLR were based on the data after the first cycle of first-line chemotherapy. ${ }^{\dagger}$, median value; ${ }^{\S}$, the patients with NLR $<3$ and NLR $\geq 3$ after the first cycle of the first-line chemotherapy were divided into lower NLR (post-chemo) and higher NLR (post-chemo) groups, respectively; * , a P value <0.05 was considered as statistically significant. CCr, creatinine clearance; NLR, neutrophil-lymphocyte ratio; UC, urothelial carcinoma.

suggested that muscle invasive bladder cancer patients with persistently high NLR after receiving neoadjuvant chemotherapy had worse prognosis (15). Notably, our study showed that a higher NLR after first cycle of first-line chemotherapy was associated with a worse prognosis for patients with UTUC.

Several researchers have contended that posttreatment NLR or changes in NLR during treatment was an efficient prognostic marker $(7,16,17)$. In lung cancer patients treated with nivolumab, non-responders had dynamic increases in NLR, whereas the NLR increase remained low in responders. Furthermore, the posttreatment NLR, but not pretreatment, was a significant predictor of prognosis (18). We speculated that a subset of patients with higher NLR before treatment might achieve good prognosis through a significant reduction of NLR from cancer treatment. However, a higher NLR not before but after the first cycle of the first-line chemotherapy was revealed as significant predictor of prognosis in this study. The result also suggested that post-treatment NLR could be an indication as to whether patients could continue and benefit from the first-line chemotherapy. We considered that post-treatment NLR could suggest the timing patients should proceed to second-line treatment, however higher NLR could be just associated with worse prognosis. In that sense, whether patients could get benefit from first-line chemotherapy should be evaluated in further study.

There are other markers that are associated with poor prognosis in urothelial carcinoma including UTUC. Tumor progression is correlated with the elevation of inflammatory cytokines, which may lead to hypoalbuminemia and increased C-reactive protein (CRP) levels (19). It was suggested that poor nutritional status and higher CRP were linked with a worse prognosis in UTUC $(20,21)$. In addition, hyponatremia and anemia are also affected by tumor mediated inflammation which could be associated with a worse prognosis $(5,22)$.

Our study has several limitations. The first is, the number of patients included in this retrospective study is relatively low. Although it was desired that all of the candidate markers were included in multivariate regression analysis, it was not possible due to limited number of patients in this study. Thus, we conducted multivariate regression analysis in model 1 and 2. The second is, there are several histology types and treatment regimens included in this study. The third is, we excluded the patients who received adjuvant therapy after surgery and had no recurrence thereafter. Thus, we could not compare NLR 
of the patients with those who experienced recurrence after adjuvant therapy. The fourth is, we set cutoff point of NLR as three based on previous studies $(6,7)$. However optimal cutoff point of NLR after the first cycle of the first-line chemotherapy should be determined from large sample sizes. Although the patients with NLR $>3$ after the first cycle of first-line chemotherapy showed poor CSS, further analysis was warranted.

\section{Conclusions}

A higher NLR after receiving the first cycle of the firstline chemotherapy could be a prognostic marker in locally advanced or metastatic UTUC patients. Although further study with larger cohorts is needed, our findings provide an indication as to whether patients could maintain their firstline chemotherapy.

\section{Acknowledgments}

Funding: None.

\section{Footnote}

Reporting Checklist: The authors have completed the REMARK reporting checklist. Available at http://dx.doi. org/10.21037/tau-21-185

Data Sharing Statement: Available at http://dx.doi. org/10.21037/tau-21-185

Conflicts of Interest: All authors have completed the ICMJE uniform disclosure form (available at http://dx.doi. org/10.21037/tau-21-185). KF serves as an unpaid editorial board member of Translational Andrology and Urology from Oct 2019 to Sep 2021. The other authors have no conflicts of interest to declare.

Ethical Statement: The authors are accountable for all aspects of the work in ensuring that questions related to the accuracy or integrity of any part of the work are appropriately investigated and resolved. The study was conducted in accordance with the Declaration of Helsinki (as revised in 2013). The study was approved by the institutional ethical review board (No. R02-247) and individual consent for this retrospective analysis was waived.

Open Access Statement: This is an Open Access article distributed in accordance with the Creative Commons Attribution-NonCommercial-NoDerivs 4.0 International License (CC BY-NC-ND 4.0), which permits the noncommercial replication and distribution of the article with the strict proviso that no changes or edits are made and the original work is properly cited (including links to both the formal publication through the relevant DOI and the license). See: https://creativecommons.org/licenses/by-nc-nd/4.0/.

\section{References}

1. Margulis V, Shariat SF, Matin SF, et al. Outcomes of radical nephroureterectomy: a series from the Upper Tract Urothelial Carcinoma Collaboration. Cancer 2009;115:1224-33.

2. Petros FG. Epidemiology, clinical presentation, and evaluation of upper-tract urothelial carcinoma. Transl Androl Urol 2020;9:1794-8.

3. Rouprêt M, Babjuk M, Compérat E, et al. European guidelines on upper tract urothelial carcinomas: 2013 update. Eur Urol 2013;63:1059-71.

4. Cheng YC, Huang CN, Wu WJ, et al. The prognostic significance of inflammation-associated blood cell markers in patients with upper tract urothelial carcinoma. Ann Surg Oncol 2016;23:343-51.

5. Fujita K, Uemura M, Yamamoto $Y$, et al. Preoperative risk stratification for cancer-specific survival of patients with upper urinary tract urothelial carcinoma treated by nephroureterectomy. Int J Clin Oncol 2015;20:156-63.

6. Taguchi S, Nakagawa T, Matsumoto A, et al. Pretreatment neutrophil-to-lymphocyte ratio as an independent predictor of survival in patients with metastatic urothelial carcinoma: a multi-institutional study. Int J Urol 2015;22:638-43.

7. Tan YG, Eu EWC, Huang HH, et al. High neutrophil-tolymphocyte ratio predicts worse overall survival in patients with advanced/metastatic urothelial bladder cancer. Int J Urol 2018;25:232-8.

8. Audenet F, Yates DR, Cussenot O, et al. The role of chemotherapy in the treatment of urothelial cell carcinoma of the upper urinary tract (UUT-UCC). Urol Oncol 2013;31:407-13.

9. Cockcroft DW, Gault MH. Prediction of creatinine clearance from serum creatinine. Nephron 1976;16:31-41.

10. Kanda Y. Investigation of the freely available easy-touse software 'EZR' for medical statistics. Bone Marrow Transplant 2013;48:452-8.

11. Kaag MG, O'Malley RL, O'Malley P, et al. Changes 
in renal function following nephroureterectomy may affect the use of perioperative chemotherapy. Eur Urol 2010;58:581-7.

12. Furubayashi N, Negishi T, Takamatsu D, et al. Timing of changing therapy from gemcitabine and cisplatin chemotherapy based on real-world data of advanced urothelial carcinoma. Oncol Lett 2020;19:2943-9.

13. Uribe-Querol E, Rosales C. Neutrophils in cancer: two sides of the same coin. J Immunol Res 2015;2015:983698.

14. Sheng IY, Diaz-Montero CM, Rayman P, et al. Blood myeloid-derived suppressor cells correlate with neutrophilto-lymphocyte ratio and overall survival in metastatic urothelial carcinoma. Target Oncol 2020;15:211-20.

15. Kaiser J, Li H, North SA, et al. The prognostic role of the change in neutrophil-to-lymphocyte ratio during neoadjuvant chemotherapy in patients with muscleinvasive bladder cancer: a retrospective, multi-institutional study. Bladder Cancer 2018;4:185-94.

16. Templeton AJ, Knox JJ, Lin X, et al. Change in neutrophilto-lymphocyte ratio in response to targeted therapy for metastatic renal cell carcinoma as a prognosticator and biomarker of efficacy. Eur Urol 2016;70:358-64.

17. Lee Y, Kim SH, Han JY, et al. Early neutrophil-tolymphocyte ratio reduction as a surrogate marker of prognosis in never smokers with advanced lung

Cite this article as: Hashimoto M, Fujita K, Nakayama T, Fujimoto S, Hamaguchi M, Nishimoto M, Kikuchi T, Adomi S, Banno E, De Velasco MA, Saito Y, Shimizu N, Mori Y, Minami T, Nozawa M, Nose K, Yoshimura K, Uemura H. Higher neutrophil-to-lymphocyte ratio after the first cycle of the first-line chemotherapy is associated with poor cancer specific survival of upper urinary tract carcinoma patients. Transl Androl Urol 2021;10(7):2838-2847. doi: 10.21037/tau-21185 adenocarcinoma receiving gefitinib or standard chemotherapy as first-line therapy. J Cancer Res Clin Oncol 2012;138:2009-16.

18. Khunger M, Patil PD, Khunger A, et al. Post-treatment changes in hematological parameters predict response to nivolumab monotherapy in non-small cell lung cancer patients. PLoS One 2018;13:e0197743.

19. Yuk HD, Ku JH. Role of systemic inflammatory response markers in urothelial carcinoma. Front Oncol 2020;10:1473.

20. Itami Y, Miyake M, Tatsumi Y, et al. Preoperative predictive factors focused on inflammation-, nutrition-, and muscle-status in patients with upper urinary tract urothelial carcinoma undergoing nephroureterectomy. Int J Clin Oncol 2019;24:533-45.

21. Fujita K, Inamoto T, Yamamoto Y, et al. Role of adjuvant chemotherapy for lymph node-positive upper tract urothelial carcinoma and the prognostic significance of C-reactive protein: a multi-institutional, retrospective study. Int J Urol 2015;22:1006-12.

22. Fujita K, Taneishi K, Inamoto T, et al. Adjuvant chemotherapy improves survival of patients with highrisk upper urinary tract urothelial carcinoma: a propensity score-matched analysis. BMC Urol 2017;17:110. 